\title{
Primary spontaneous pneumothorax after exposure to very loud music
}

\author{
Krivokuca $\mathbf{I}^{* *}$ and Lammers JWJ ${ }^{2}$ \\ ${ }^{1}$ Department of Respiratory Medicine, MC Zuiderzee Hospital Lelystad, Ziekenhuisweg 100, 8233 AA Lelystad, The Netherlands \\ ${ }^{2}$ Department of Respiratory Medicine, Division of Heart \& Lungs, University Medical Centre Utrecht, Heidelberglaan 100, 3584 CX, Utrecht, The Netherlands
}

\begin{abstract}
Primary spontaneous pneumothorax is relatively often diagnosed in the emergency departments especially in tall, thin, young, cigarette smokers. It is generally believed and earlier reported by Noppen in 2004, that primary spontaneous pneumothorax could develop after exposure to very loud music. We briefly describe here a young healthy male with a primary spontaneous pneumothorax shortly after listening to very loud music.
\end{abstract}

\section{Introduction}

Pneumothorax is the presence of air between the visceral and the parietal pleura of the lung [1-5]. Jean Marc Gaspard Itard, a student of Laennec, described five tuberculosis patients with pneumothorax in 1803 and introduced the word pneumothorax [6]. Pneumothoraces are usually subdivided into traumatic pneumothoraces which are the result of a chest trauma and non-traumatic (spontaneous) pneumothoraces which develop without preceding chest trauma. "Iatrogenic pneumothoraces" are a subtype of traumatic pneumothoraces which occur during or after certain diagnostic or therapeutic procedures like inserting a central line in the subclavian artery. Non-traumatic (spontaneous) pneumothoraces are subdivided into primary spontaneous pneumothorax when pulmonary parenchyma is apparently normal and secondary spontaneous pneumothorax which may occur after rupture of intrapulmonary bullae or blebs. Here we describe the occurrence of a primary spontaneous pneumothorax in a young man after listening to very loud music.

\section{Case report}

An 18-year-old healthy thin male with a half year history of smoking, presented to the emergency department with a left sided chest pain without dyspnoea, coughing or mucus production and without antiphospholipid syndrome or other clotting abnormalities in the past. He denied any preceding chest trauma, but his symptoms developed on his way home after a pop concert where he had been listened to very loud music while standing in the front row. His respiratory rate was 18 breaths/min and his $\mathrm{SpO}_{2}$ was $96 \%$ breathing room air. He was afebrile and his pulse rate was 60 beats per minute. Furthermore, he was normotensive, and examination of the lungs revealed normal vesicular breath sounds, no wheezing or rhonchi and his percussion was normal. Laboratory results and his ECG were unremarkable. A chest $\mathrm{x}$-ray was performed (Figure 1) which showed a left sided pneumothorax. We admitted this young man for one night to observe his symptoms and the size of the pneumothorax. After conservative treatment without removing air with a needle or a chest tube drainage his symptoms improved and the size of the pneumothorax on the chest $\mathrm{x}$-ray remained stable. Several days after his discharge, he visited our outpatient clinic

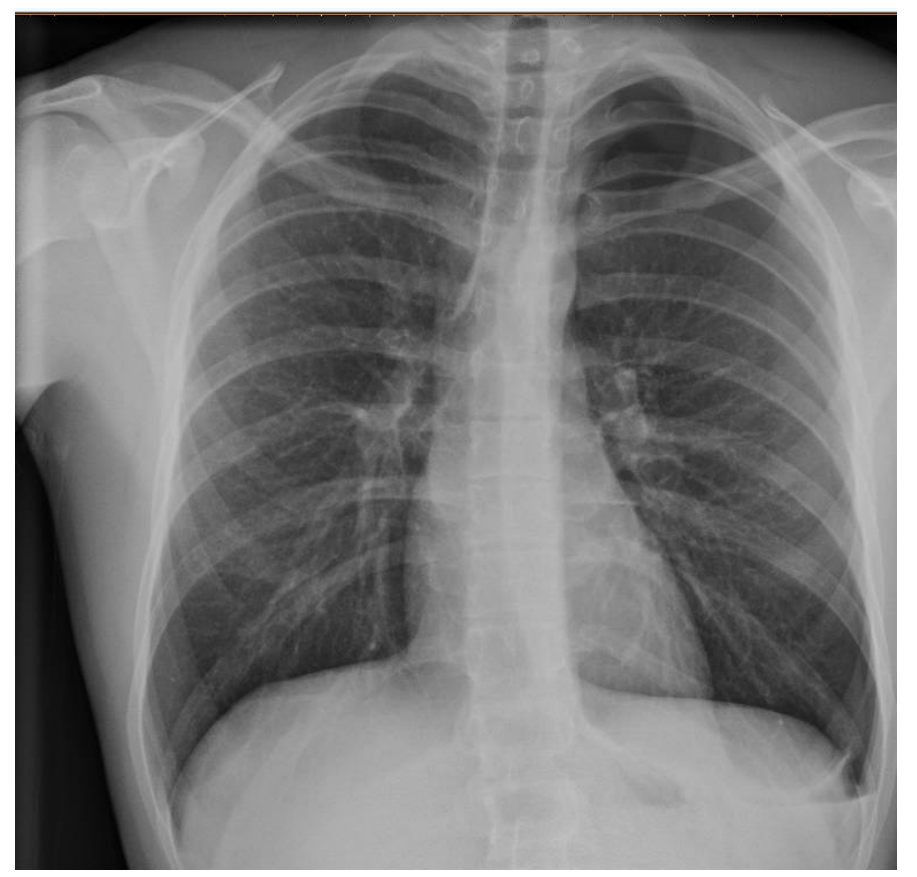

Figure 1. Chest radiograph on admission

and the pneumothorax on the chest $\mathrm{x}$-ray had completely resolved. We did check his alpha-1 antitripsin level to exclude alpha-1 antitrypsin deficiency which appeared to be normal. He did stop smoking and luckily, he did not have a recurrence of his pneumothorax.

${ }^{*}$ Correspondence to: Krivokuca I, Department of Respiratory Medicine, MC Zuiderzee Hospital Lelystad, 8233 AA Lelystad, The Netherlands, Tel: 0031320271911,0031887556180; E-mail: sciencia0@yahoo.com

Received: May 12, 2019; Accepted: May 23, 2019; Published: May 27, 2019 


\section{Discussion}

Noppen reported four patients with primary spontaneous pneumothorax after exposure to very loud music. He hypothesised that sound pressure waves could result in pressure differences and a tear of the alveolar wall or the wall of bullae or blebs [2].

The incidence of non-traumatic, primary spontaneous pneumothorax is much higher in males than in females (7.4 to 18 per 100.000 males per year, compared to 1.2 to 6 per 100.000 females per year) [4]. The symptoms and signs of primary spontaneous pneumothorax vary depending on the size of the pneumothorax. A small pneumothorax, with the distance between the chest wall and lung of less than $2 \mathrm{~cm}$ on a chest $\mathrm{x}$-ray, could be asymptomatic, while larger pneumothoraces with a distance larger than $2 \mathrm{~cm}$ are usually symptomatic with symptoms such as chest pain and dyspnea, which are the presenting symptoms in 64 to $85 \%$ of pneumothoraces [1]. Smokers have a much higher lifetime risk of developing a pneumothorax $(12 \%$ vs. $0.1 \%)$. Primary spontaneous pneumothorax has a recurrence rate between 20 and $60 \%$ in the first 3 years after the first episode of pneumothorax [1] and stopping smoking could substantially reduce the risk of pneumothorax.

\section{Conclusion}

Although primary spontaneous pneumothorax after listening to loud music is rarely reported, it may well be a life-threatening disorder that should be considered in all healthy young patients with chest pain or dyspnea occurring after exposure to loud music.

\section{References}

1. Sajadi-Ernazarova KR, Gupta R (2019) Acute Pneumothorax Evaluation and Treatment. StatPearls [Crossref]

2. Noppen M, Verbanck S, Harvey J, VanHerreweghe R, Meysman M, et al. (2004) Music: a new cause of primary spontaneous pneumothorax. Thorax 59: 722-724. [Crossref]

3. Haynes D, Baumann MH (2011) Pleural controversy: aetiology of pneumothorax. Respirology 16: 604-610. [Crossref]

4. Ganaie MB, Maqsood U, Lea S, Bankart MJ, Bikmalla S, et al. (2019) How should complete lung collapse secondary to primary spontaneous pneumothorax be managed? Clin Med (Lond) 19: 163-168. [Crossref]

5. Bintcliffe O, Maskell N (2014) Spontaneous Pneumothorax. BMJ 348: g2928. [Crossref]

6. Light RW, Lee YCG (2008) Textbook of Pleural Diseses. Second Edition.

Copyright: (C2019 Krivokuca I. This is an open-access article distributed under the terms of the Creative Commons Attribution License, which permits unrestricted use, distribution, and reproduction in any medium, provided the original author and source are credited. 